\title{
AN EXPLICIT DYNAMICS APPROACH TO THE SIMULATION OF CRACK PROPAGATION IN THIN SHELLS USING REDUCED INTEGRATION SOLID-SHELL ELEMENTS
}

\author{
M. Pagani, U. Perego \\ Department of Structural Engineering, Politecnico di Milano (umberto.perego@polimi.it)
}

\begin{abstract}
Fracture propagation in laminated shell structures, due to impact or cutting, is a highly nonlinear problem which is more conveniently simulated using explicit finite element approaches. Solid-shell elements are better suited for the discretization in the presence of complex material behavior and delamination, since they allow for a correct representation of the through the thickness stress. In the presence of cutting problems with sharp blades, classical crack-propagation approaches based on cohesive interfaces may prove inadequate. New "directional" cohesive interface elements are here proposed to account for the interaction with the cutter edge. The element small thickness leads to very high eigenfrequencies, which imply overly small stable time-steps. A new selective mass scaling technique is here proposed to increase the time-step without affecting accuracy.
\end{abstract}

Keywords: Cutting, Explicit Dynamics, Crack Propagation, Mass Scaling, Solid-Shell Elements.

\section{INTRODUCTION}

Finite element simulation of crack propagation in shell structures is a timely topic in computational mechanics $[1,2,3,4,5]$. The particular case of fracture initiated by contact against a sharp blade deserves a specific attention [6]. In view of the high nonlinearity of the problem, due to contact, plasticity, large strains, fracture initiation, crack propagation and possible delamination, explicit dynamics simulations are generally preferred. An extremely fine mesh may be required to resolve the blade tip curvature radius and to avoid interferences with the process zone in the case that cohesive interface elements are used. Complex material behavior, due to large strain plasticity and delamination requires an accurate description of through the thickness stresses. For this reason, solid-shell elements rather than classical shellelements are often used. On the other hand, the simulation of complex material evolutions (large strains, plasticity, delamination), to be enforced at Gauss points, entails significant computational costs which suggest the adoption of reduced integration with hourglass control. The conditional stability of explicit simulations, requiring small time-steps, also contributes to increasing the computing burden. It is therefore particularly important that all operations at the element level are carried out in a computationally effective way and that the stable time-step is as large as possible.

In the present paper, some of the above mentioned computational issues are addressed. The issue of the time-step size is tackled by means of the introduction of a selective mass scaling technique, specifically conceived for solid-shell elements. A computationally inexpensive 
linear transformation of the element degrees of freedom, allows to scale only masses connected to higher order element modes, while leaving those associated to rigid body modes unaltered. This provision is shown to lead to substantial saving in computational costs, with stable time-steps which can be of one to two orders of magnitude larger and with only marginal accuracy loss. As for the simulation of the cutting of thin shells, the methodology recently proposed in [7] is here implemented in combination with shell and solid-shell elements of the type proposed in [8]. The methodology is based on the notion of directional cohesive elements and is specially conceived for the simulation of crack development due to cutting with a sharp blade.

\section{SELECTIVE MASS SCALING}

\subsection{Selective mass scaling procedure}

In solid-shell elements, the fact that the thickness dimension is always significantly smaller than the in-plane dimensions leads to a very high finite element maximum eigenfrequency. This is particularly relevant when explicit time integration is used in dynamic analyses, since the stable time-step size decreases with the maximum among element eigenfrequencies. This problem can be circumvented by adopting a mass scaling technique, whereby masses are increased so as to reduce the element maximum eigenfrequency.

Since individual finite elements contribute to the lowest structural eigenmodes mainly with the inertia associated to their rigid body modes, in inertia dominated problems more accurate results can be obtained by selectively scaling element masses, in such a way that masses associated to element rigid body modes are not modified. A theoretically motivated scaling, which satisfies this requisite, can be obtained by summing to the mass matrix the stiffness matrix multiplied by a scaling parameter $[9,10]$. This scaling can be shown to selectively reduce the higher structural eigenfrequencies, with little or zero modifications of the lowest ones. The price to pay is that, after the scaling, the originally lumped mass matrix becomes non-diagonal, which is a serious computational drawback in explicit dynamics. To avoid this problem, the technique proposed in this paper is based on a linear transformation of the solid-shell element nodal degrees of freedom, which allows to selectively apply the mass scaling while preserving the mass lumping. This can be accomplished in a simple and computationally inexpensive way, so that the time-step size can be shown to be governed almost exclusively by the element in-plane dimensions (element in-plane traversal time), independent of the element thickness.

The dynamic equilibrium equations of the undamped discretized system are given by

$$
\mathbf{M a}+\mathbf{f}^{i n t}=\mathbf{f}^{e x t}
$$

where $\mathbf{a}$ is the vector of nodal accelerations, $\mathbf{M}$ is the mass matrix, $\mathbf{f}^{\text {int }}$ and $\mathbf{f}^{\text {ext }}$ the vectors of equivalent internal and external nodal forces, respectively. The effect of prescribed displacements is assumed to be incorporated in $\mathbf{f}^{\text {ext }}$. The implementation of the central difference integration scheme requires that the accelerations are computed at each time-step as

$$
\mathbf{a}=\mathbf{M}^{-1} \mathbf{f}
$$




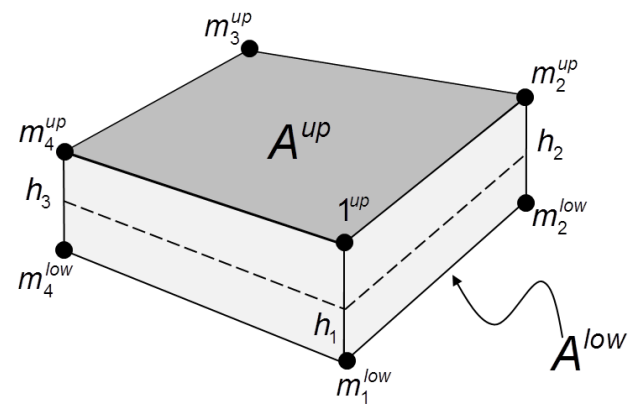

Figure 1. Solid-shell element: definition of upper and lower surfaces.

where $\mathbf{f}=\mathbf{f}^{\text {ext }}-\mathbf{f}^{\text {int }}$ and for effective computations $\mathbf{M}$ is assumed to be diagonal.

Making reference to the eight-node solid-shell element with lumped masses shown in Figure 1, the upper and lower surfaces of the element can be easily identified. If $\mathbf{a}_{i}^{u p}$ and $\mathbf{a}_{i}^{\text {low }}, i=1, \ldots 4$, denote the accelerations of corresponding nodes on the two surfaces, the accelerations $\mathbf{a}_{i}^{a v e}$ governing the element rigid body modes can be defined using the following linear transformation

$$
\mathbf{a}_{i}^{a v e}=\frac{\mathbf{a}_{i}^{u p}+\mathbf{a}_{i}^{\text {low }}}{2}
$$

while the accelerations $\mathbf{a}_{i}^{\text {diff }}$, governing the higher order modes, are defined as

$$
\mathbf{a}_{i}^{\text {diff }}=\frac{\mathbf{a}_{i}^{u p}-\mathbf{a}_{i}^{\text {low }}}{2}
$$

with the inverse transformations

$$
\mathbf{a}_{i}^{u p}=\mathbf{a}_{i}^{\text {ave }}+\mathbf{a}_{i}^{\text {diff }}, \quad \mathbf{a}_{i}^{\text {low }}=\mathbf{a}_{i}^{\text {ave }}-\mathbf{a}_{i}^{\text {diff }}
$$

In matrix form, one can write for element $e$

$$
\underset{24 \times 1}{\mathbf{a}^{e}}=\left\{\begin{array}{c}
\mathbf{a}^{u p} \\
12 \times 1 \\
\mathbf{a}_{12 \times 1}^{l o w}
\end{array}\right\}^{e}=\mathbf{T} \hat{\mathbf{a}}^{e}=\left[\begin{array}{cc}
\mathbf{I} & \mathbf{I} \\
\mathbf{I} & -\mathbf{I}
\end{array}\right]^{e}\left\{\begin{array}{c}
\mathbf{a}^{\text {ave }} \\
12 \times 1 \\
\mathbf{a}^{\text {diff }} \\
12 \times 1
\end{array}\right\}^{e}, \quad \hat{\mathbf{M}}^{e}=\mathbf{T}^{T} \mathbf{M}^{e} \mathbf{T}, \quad \hat{\mathbf{f}}^{e}=\mathbf{T}^{T} \mathbf{f}^{e}=\left\{\begin{array}{c}
\mathbf{f}^{\text {ave }} \\
\mathbf{f}^{\text {diff }}
\end{array}\right\}^{e}
$$

where $\mathbf{I}$ is the $12 \times 12$ identity matrix. In (6), a superposed hat denotes vectors and matrices expressed in terms of average and difference degrees of freedom.

For distorted elements, $\hat{\mathbf{M}}^{e}$ is in general not diagonal even when $\mathbf{M}^{e}$ is lumped. A diagonal mass matrix $\hat{\mathbf{M}}_{\text {lumped }}^{e}$ can be easily obtained e.g. using the HRZ lumping procedure [11]

$$
\hat{\mathbf{M}}_{\text {lumped }}^{e}=\left[\begin{array}{cc}
\mathbf{M}_{12 \times 12}^{\text {ave }} & \mathbf{0} \\
\mathbf{0} & \underset{12 \times 12}{\mathbf{M}^{\text {diff }}}
\end{array}\right]^{e}=\left[\begin{array}{ccc|ccc}
\ddots & & \mathbf{0} & & & \\
& \hat{\mathbf{m}}_{i} & & & \mathbf{0} & \\
\mathbf{0} & & \ddots & & & \\
\hline & & & \ddots & & \mathbf{0} \\
& \mathbf{0} & & & \hat{\mathbf{m}}_{i} & \\
& & & \mathbf{0} & & \ddots
\end{array}\right]^{e}
$$




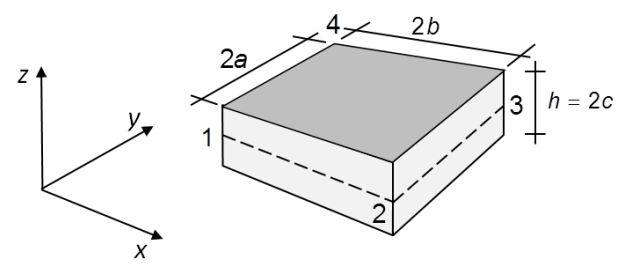

Figure 2. Reference element for analytical computation of maximum eigenfrequency.

where

$$
\hat{\mathbf{m}}_{i}^{e}=\left[\begin{array}{ccc}
\hat{m}_{i} & 0 & 0 \\
0 & \hat{m}_{i} & 0 \\
0 & 0 & \hat{m}_{i}
\end{array}\right]^{e}, \quad i=1, \ldots 4
$$

If the original mass matrix $\mathbf{M}^{e}$ is already lumped, one simply has $\hat{m}_{i}=m_{i}^{u p}+m_{i}^{\text {low }}$.

At this point, the element maximum eigenfrequency can be scaled down, without affecting the element rigid body modes, leaving $\mathbf{M}^{\text {ave }}$ unaltered and multiplying $\mathbf{M}^{\text {diff }}$ by a scale factor $\alpha>1$ defined as

$$
\alpha=\beta \frac{A^{\text {up }}+A^{\text {low }}}{2 h^{2}}, \quad \hat{\overline{\mathbf{M}}}_{\text {lumped }}^{e}=\left[\begin{array}{cc}
\mathbf{M}^{\text {ave }} & \mathbf{0} \\
\mathbf{0} & \alpha \mathbf{M}^{\text {diff }}
\end{array}\right]^{e}
$$

where $A^{u p}$ and $A^{\text {low }}$ are the areas of the element upper and lower surfaces and $h=\frac{1}{4} \sum_{i=1}^{4} h_{i}$ is the average element thickness, $h_{i}$ being the distance between corresponding nodes on the upper and lower surfaces. The factor $\beta$ can be adjusted in such a way that the scaled critical time-step size approaches the in-plane element traversal time.

To obtain a reduction of the maximum eigenfrequency, the new element degrees of freedom with scaled masses need to be used also for the global structure. This is not a problem since they are nodal degrees of freedom and can be assembled with the usual procedure. If the transformed element matrices are assembled in the equation of motion, one simply obtains

$$
\hat{\bar{M}} \hat{\bar{a}}=\hat{\mathbf{f}}
$$

where a superposed bar denotes the scaled mass matrix and relative nodal accelerations. Referring to the $j-t h$ node in the mesh, once $\overline{\mathbf{a}}_{j}^{\text {ave }}$ and $\overline{\mathbf{a}}_{j}^{\text {diff }}$ have been computed as

$$
\overline{\mathbf{a}}_{j}^{\text {ave }}=\frac{\mathbf{f}_{j}^{\text {ave }}}{\hat{m}_{j}}=\frac{\mathbf{f}_{j}^{u p}+\mathbf{f}_{j}^{\text {low }}}{m_{j}^{u p}+m_{j}^{\text {low }}}, \quad \overline{\mathbf{a}}_{j}^{\text {diff }}=\frac{\mathbf{f}_{j}^{\text {diff }}}{\alpha \hat{m}_{j}}=\frac{\mathbf{f}_{j}^{u p}-\mathbf{f}_{j}^{\text {low }}}{\alpha\left(m_{j}^{u p}+m_{j}^{\text {low }}\right)}
$$

one can easily recover the nodal accelerations in terms of the original degrees of freedom

$$
\begin{gathered}
\overline{\mathbf{a}}_{j}^{u p}=\overline{\mathbf{a}}_{j}^{\text {ave }}+\overline{\mathbf{a}}_{j}^{\text {diff }}=\frac{(\alpha+1) \mathbf{f}_{j}^{u p}+(\alpha-1) \mathbf{f}_{j}^{\text {low }}}{\alpha\left(m_{j}^{u p}+m_{j}^{\text {low }}\right)} \\
\overline{\mathbf{a}}_{j}^{\text {low }}=\overline{\mathbf{a}}_{j}^{\text {ave }}-\overline{\mathbf{a}}_{j}^{\text {diff }}=\frac{(\alpha-1) \mathbf{f}_{j}^{u p}+(\alpha+1) \mathbf{f}_{j}^{\text {low }}}{\alpha\left(m_{j}^{u p}+m_{j}^{\text {low }}\right)}
\end{gathered}
$$

The simple expressions of the nodal accelerations in (12) show that the mass scaling can be applied without actually implementing the variable transformation in (6). The modified nodal 
acceleration values can be easily obtained from nodal quantities defined in a standard way, which implies that an existing code requires only minimal modifications.

A conceptually almost identical scaling procedure was presented in [12]. In that case, rather than to the masses, the scaling was applied directly to nodal accelerations. If $m_{j}^{u p}=$

$m_{j}^{\text {low }}$, the definition of $\mathbf{a}_{j}^{\text {diff }}$ obtained according to the procedure proposed in [12], is coincident with the definition (4) given here. In this case the two scaling methods are therefore identical. The main advantage of the procedure proposed here is that it provides a consistent variable transformation also for the stiffness matrix, so allowing for the analytical computation of $\omega_{\text {max }}^{e}$. This can be achieved in closed form for elements of parallelepiped shape as follows. Compute the coefficients ( $a, b, c$ being the element semi-dimensions, see Figure 2 )

$$
\begin{aligned}
& \gamma=\frac{c}{a} ; \quad \lambda=\frac{c}{b} \\
& C_{0}=-144^{3} \gamma \lambda(1+\nu) \\
& C_{1}=144^{2}\left(\lambda^{2}+\gamma^{2}+\alpha \gamma^{2} \lambda^{2}\right) \\
& C_{2}=-144 \gamma \lambda\left[1+\alpha\left(\gamma^{2}+\lambda^{2}\right)\right](1-\nu) \\
& C_{3}=\alpha \gamma^{2} \lambda^{2}(1-2 \nu) \\
& p=\frac{C_{1}}{C_{3}}-\frac{1}{3}\left(\frac{C_{2}}{C_{3}}\right)^{2} \\
& q=\frac{C_{0}}{C_{3}}+\frac{2}{27}\left(\frac{C_{2}}{C_{3}}\right)^{2}-\frac{1}{3} \frac{C_{1} C_{2}}{C_{3}^{2}}
\end{aligned}
$$

Check discriminant. If

$$
\left(\frac{q}{2}\right)^{2}+\left(\frac{p}{3}\right)<0
$$

then the maximum eigenfrequency is real and is given by

$$
\Delta t=0.8 \frac{2}{\bar{\omega}_{\max }}=0.8\left(24 \sqrt{\frac{a b \rho(1+\nu)}{\eta E}}\right)
$$

where

$$
\varphi=\arccos \left(-\frac{q}{2 \sqrt{-\left(\frac{p}{3}\right)^{3}}}\right) ; \quad \eta=2 \sqrt{-\frac{p}{3}} \cos \left(\frac{\varphi}{3}\right)-\frac{1}{3} \frac{C_{2}}{C_{3}}
$$

$\rho$ is the mass density and 0.8 is a reduction coefficient intended to provide a safety margin for stability in the nonlinear case. In the case of slightly distorted elements, an estimate of the stable time-step can be obtained by computing the maximum eigenfrequency of the largest parallelepiped contained in the distorted element.

\subsection{Application to a cylindrical shell hinged on two sides}

A cylindrical shell hinged on two sides and free on the other two sides, subjected to a transversal concentrated center load, has been analyzed using the $16 \times 16$ SHB8PS (see [13]) solid-shell element mesh shown in Figure 3. The problem has been used in [14] to test the application of the scaled director conditioning technique [15] in explicit dynamics simulations. The shell has a radius of 5 meters, a thickness of 0.01 meters and a center 


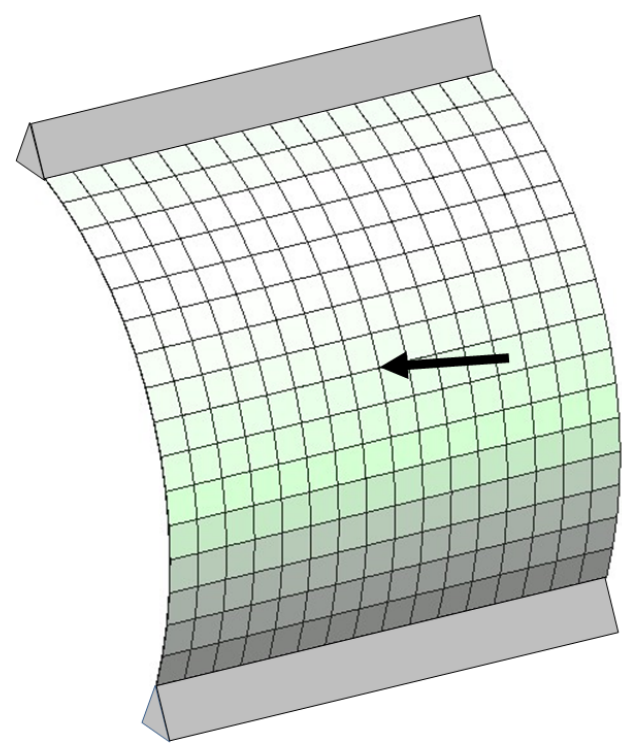

Figure 3. Cylindrical shell hinged on two sides: finite element mesh, boundary conditions and load.

opening angle of $80^{\circ}$. The material is assumed to be linear elastic with a Young's modulus of $2.0 \cdot 10^{9} \mathrm{~N} / \mathrm{m}^{2}$, Poisson's ratio 0.0 and mass density $1.0 \cdot 10^{5} \mathrm{~kg} / \mathrm{m}^{3}$. The load is applied with a linear ramp from a zero initial value to $1 \mathrm{MN}$ after 0.2 seconds, and then kept constant.

The analysis has been carried out using three different scaling factors $\alpha: \alpha=1.27 \cdot 10^{2}$, $\alpha=1.27 \cdot 10^{3}$ and $\alpha=1.27 \cdot 10^{4}$ (see equation 9 ). A reference analysis has also been carried out with the finite element code Abaqus, using a $32 \times 32$ mesh of fully integrated shell elements (S4 element type from Abaqus element library). The results in terms of center point displacement evolution are shown in Figure 4.

The center point displacement evolutions for the unscaled and the $\alpha=1.27 \cdot 10^{2}$ analyses are very similar for the whole analysis duration. With $\alpha=1.27 \cdot 10^{3}$ there is good agreement up to the peak displacement. After the peak, the analysis with $\alpha=1.27 \cdot 10^{3}$ exhibits a displacement reduction, which is not observed in the previous curves. It should be noted however that the same displacement reduction is exhibited by the Abaqus reference curve, which remains close to the $\alpha=1.27 \cdot 10^{3}$ curve throughout the analysis. The last curve, with $\alpha=1.27 \cdot 10^{4}$, diverges significantly from the others, meaning that the adopted mass scaling affects in an unacceptable way the shell dynamic response. Snapshots of the shell deformation are shown in Figure 5 for the case $\alpha=1.27 \cdot 10^{3}$. Another beneficial effect of the mass scaling can be further observed noting that the $\alpha=1.27 \cdot 10^{3}$ curve is smoother than the unscaled curve, meaning that the spurious higher frequencies are reduced as a consequence of the adopted mass scaling.

The initial time step-size used with the different analyses is reported in Table 1. While in the unscaled case the time-step is computed using Gershgorin upper bound, in the scaled cases it is computed using the analytical procedure illustrated in the previous section. In fact, Gershgorin bound cannot be used when mass scaling is applied, since it returns the same value $\Delta t_{\text {Gershgorin }}=4.64 \cdot 10^{-4}$ for all the adopted values of $\alpha$. From Table 1 , it can be observed that the $\alpha=1.27 \cdot 10^{3}$ mass scaling provides a gain of almost two orders of magnitude with 
Table 1. Cylindrical shell hinged on two sides: initial time-step size.

\begin{tabular}{|c|c|c|c|c|c|}
\cline { 2 - 6 } \multicolumn{1}{c|}{} & Unscaled & $\alpha=1.27 \cdot 10^{2}$ & $\alpha=1.27 \cdot 10^{3}$ & $\alpha=1.27 \cdot 10^{4}$ & Abaqus-S4 \\
\hline$\Delta t[\mathrm{sec}]$ & $6.71 \cdot 10^{-5}$ & $7.96 \cdot 10^{-4}$ & $2.52 \cdot 10^{-3}$ & $3.09 \cdot 10^{-3}$ & $\approx 1 . \cdot 10^{-5}$ \\
\hline
\end{tabular}

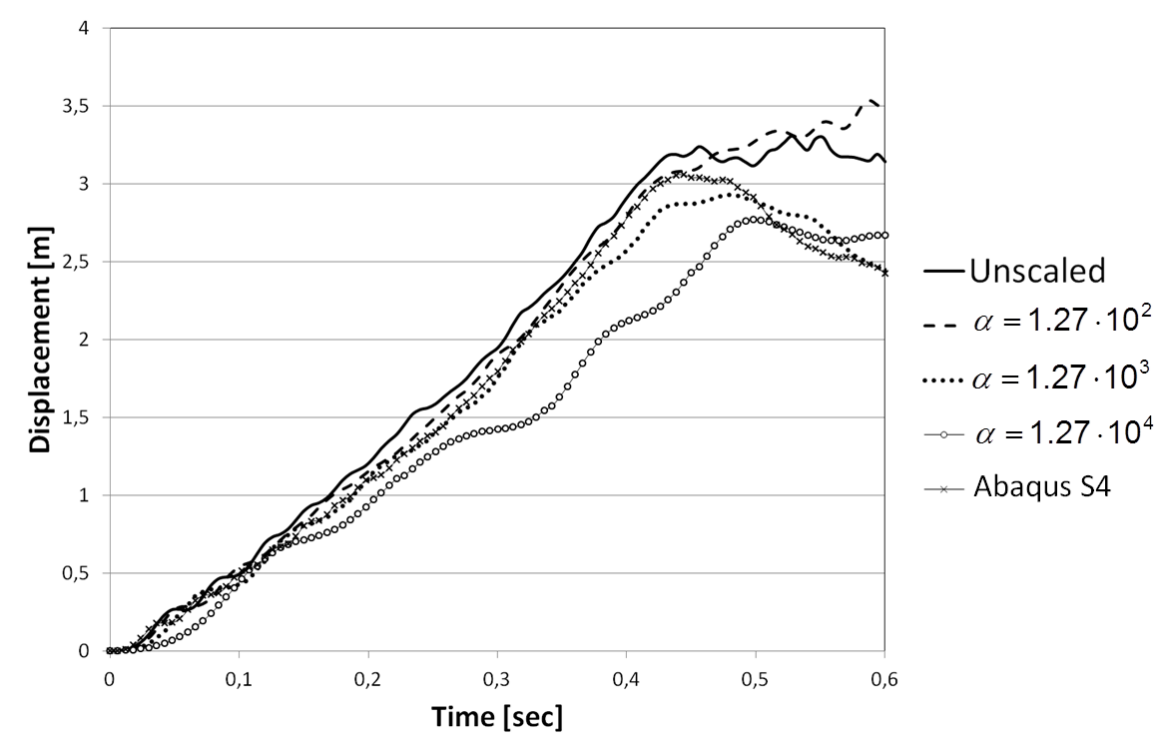

Figure 4. Cylindrical shell hinged on two sides: time evolution of center point deflection with scaled and unscaled masses, for different scaling factors.

respect to the unscaled analysis. The Abaqus time-step is also significantly smaller, but one should take into account that the Abaqus mesh is made of elements that are two times smaller than those used in the $\alpha=1.27 \cdot 10^{3}$ analysis. On the other hand, the additional gain obtained with $\alpha=1.27 \cdot 10^{4}$ is relatively small, and does not compensate for the accuracy loss.

\section{3. "DIRECTIONAL” COHESIVE ELEMENTS FOR THE SIMULATION OF CRACK PROPAGATION DUE TO CUTTING}

In finite element approaches to fracture, the propagating discontinuity is often modeled by introducing a cohesive interface between adjacent shell elements wherever a prescribed propagation criterion is exceeded at a node [1]. In this case, opposite cohesive forces are introduced across the discontinuity, their direction depending only on the direction of the displacement jump and on the adopted cohesive law. When the material is quasi-brittle and/or the impacting object is blunt, there is no interference between the object and the cohesive region, because the ultimate cohesive opening displacement is much smaller than the typical size of the object. On the contrary, when the material is very ductile, or the cutting blade is sharp, it may well happen that the blade intersects the trajectory of the cohesive forces (Figure 6), giving rise to inaccurate predictions of the crack propagation. This problem does not occur when crack propagation is simulated by removing damaged elements from the mesh, as it is currently done in advanced commercial finite element codes. In this case the contact algorithm is active on the element until the element is removed and penetration of the blade is not allowed. However, this approach requires a mesh of the shell body fine enough to conform 

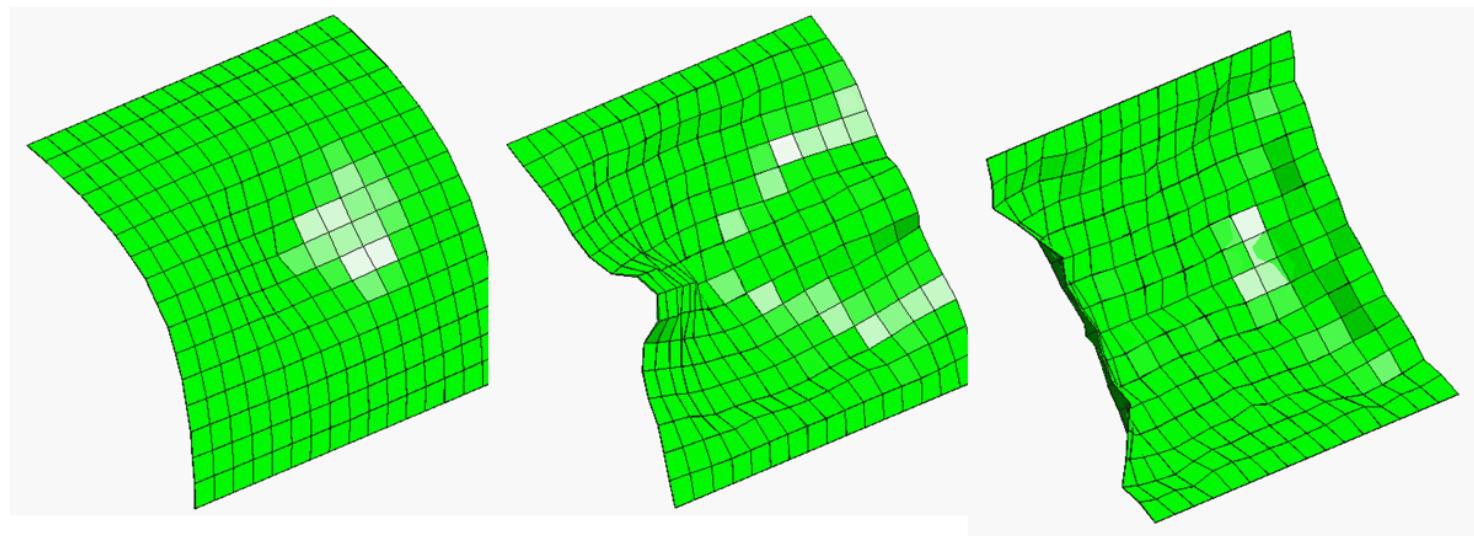

Figure 5. Cylindrical shell hinged on two sides: snapshots showing snap-through configurations.

to the blade edge.

A new type of "directional" cohesive interface element, to be placed at the interface between adjacent shell elements, has been developed in [7] for the simulation of blade cutting of thin shells. In these elements, the cohesive forces acting on the two opposite sides of the crack can have different directions whenever the cohesive region is crossed by the cutting blade.

When the selected fracture criterion is met at a given node, the node is duplicated and it is assumed that cohesive forces are transmitted between the newly created pair of nodes by a massles "cable", i.e. a truss element introduced ad hoc in the model in correspondence of each pair of separating nodes. These cables are geometric entities, whose main purpose is to detect contact against the cutting blade. They are initially straight segments, naturally endowed with a length, which is simply given by the distance between the nodes, and by a tension-elongation softening law, which accounts for the cohesive behavior. When a point of a cable element is detected to be in contact with the blade, the cable element is subdivided into two elements by introducing a joint in correspondence of the contact point, which is forced to move along the cutting edge of the cutter (Figure 7). The length of the cable is now defined as the sum of the lengths of the two constituent elements and the cohesive force is defined to be inversely proportional to the cable current total length, according to the assumed cohesive law.
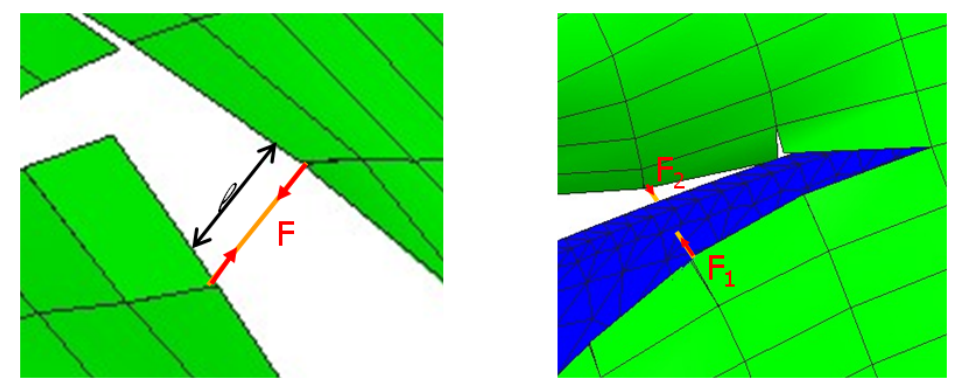

Figure 6. Classical cohesive interfaces: interference between cohesive process zone and cutting blade. 
a)

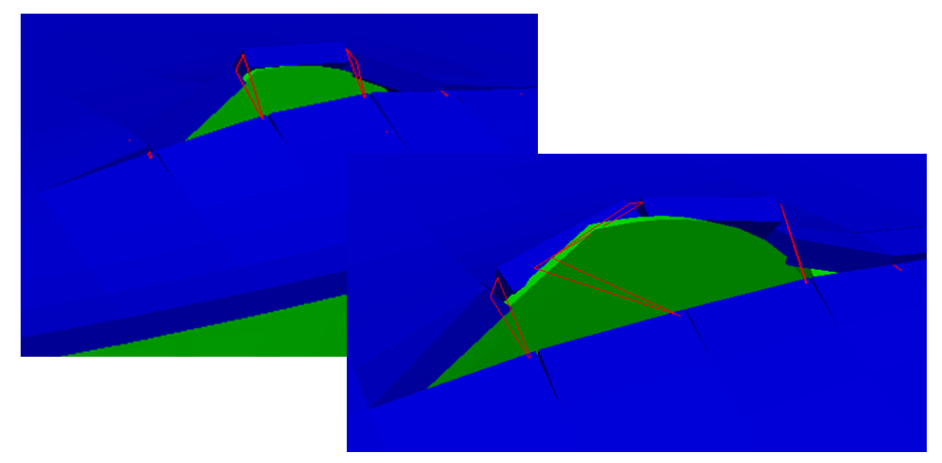

b)

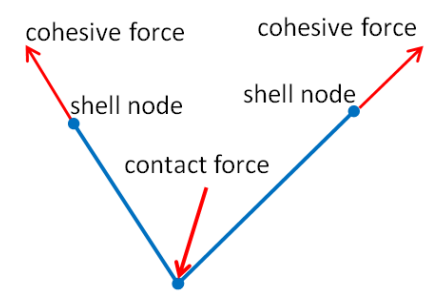

Figure 7. Directional cohesive interfaces: a) interaction between cohesive process zone and cutting blade; b) transmission of cohesive forces to crack separating sides.

In the present contribution, the proposed approach has been implemented in conjunction with the solid-shell element of Schwarze and Reese [8] and has been applied to the cutting of the elastic circular thin shell with a rotating blade shown in Figure 8, which was analyzed in [7] using shell elements of the MITC4 type [16]. The results, expressed in terms of applied torque vs. blade rotation, show that this type of elements can produce accurate results. The figure shows the used mesh of solid-shell elements and a snapshot of the analyses where the interaction between the blade and the cohesive process zone between separating solid-shell elements can be clearly appreciated. The plot shows a comparison between the results obtained in [16] with classical shell elements (dark blue curve) and the results obtained here (light blue curve). Both analyses compare well with the experimental results (black curve). The discrepancy in the initial part of the plot is due to two reasons. At the beginning of the analysis, the cutting teeth are not contacting the sheet and therefore a zero resisting torque is computed in the numerical analyses. In reality, the rotating cap has to win the resisting friction due to the contact with the screwing system, which is not modeled here. Once contact with the cutting blade is established, the shell deforms elastically until the fracture criterion is met for the first time (no plasticity in the shell core is included in the model). This explains the steep slope in the initial response of the numerical curves, which does not account for the shell elastoplastic deformation preceding fracture initiation.
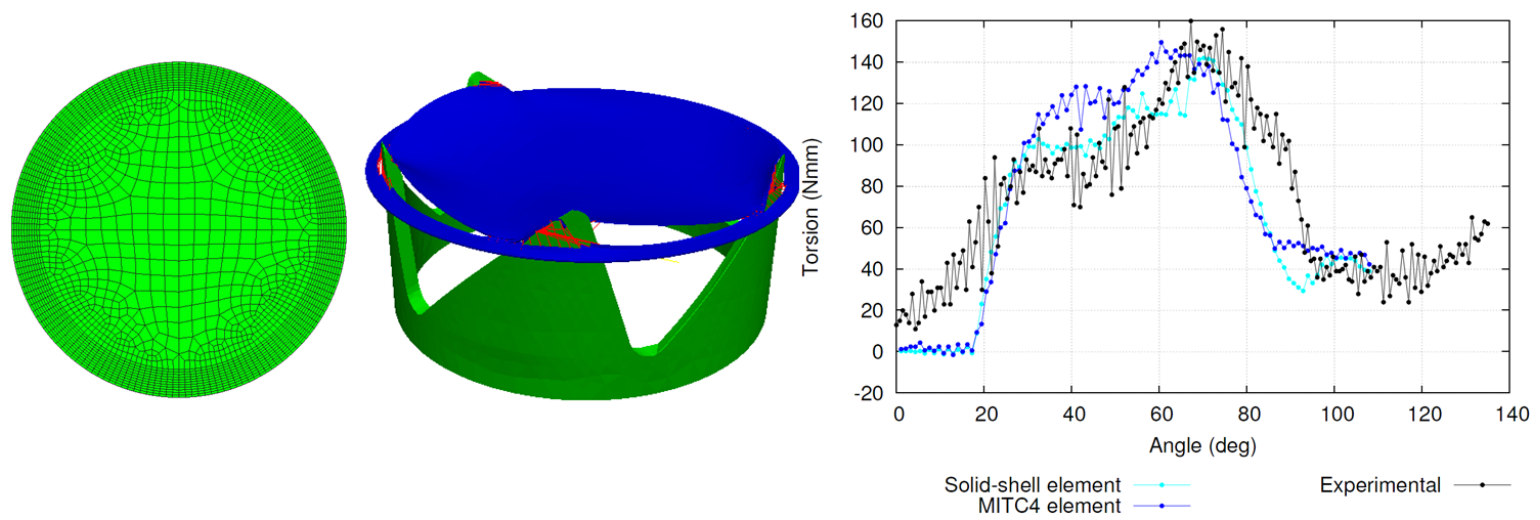

Figure 8. Blade cutting of a thin shell: comparison between experimental and numerical torque vs. rotation curves. 


\section{CONCLUSIONS}

In the present paper, two issues concerned with the simulation of the blade cutting of thin shells have been addressed. First, a selective mass scaling technique, specifically conceived for solid-shell elements has been proposed with the purpose of increasing the element maximum stable time-step in explicit dynamics simulations, without affecting the global structural response in inertia dominated problems. The scaling procedure merely consists of a modification of nodal accelerations and therefore requires only minimal modifications of existing codes. Closed form formulas for the consistent analytical derivation of the element maximum eigenfrequency and, hence, for an accurate estimate of the critical time-step size have also been provided for the case of parallelepiped elements. The procedure can also be easily extended to slightly distorted elements. Extension to high distortion is currently under study. Second, a "directional" interface cohesive element has been developed to correctly account for the interaction between the sharp cutting blade and the cohesive process zone. The simulation of a cutting experiment has shown that the proposed approach can accurately reproduce the physical process.

\section{References}

[1] F. Cirak, M. Ortiz, and A. Pandolfi, "A cohesive approach to thin-shell fracture and fragmentation," Computer Methods in Applied Mechanics and Engineering, vol. 194, no. 21-24, pp. 2604-2618, 2005.

[2] P. M. A. Areias and T. Belytschko, "Non-linear analysis of shells with arbitrary evolving cracks using xfem," International Journal for Numerical Methods in Engineering, vol. 62, no. 3, pp. 384-415, 2005.

[3] P. M. A. Areias, J. H. Song, and T. Belytschko, "Analysis of fracture in thin shells by overlapping paired elements," Computer Methods in Applied Mechanics and Engineering, vol. 195, no. 41-43, pp. 5343-5360, 2006.

[4] P. D. Zavattieri, "Modeling of crack propagation in thin-walled structures using a cohesive model for shell elements," Journal of Applied Mechanics, vol. 73, no. 6, pp. 948-958, 2006.

[5] J. H. Song and T. Belytschko, "Dynamic fracture of shells subjected to impulsive loads," Journal of Applied Mechanics, vol. 76, no. 5, pp. 051301_1-051 301_9, 2009.

[6] T. Atkins, The science and engineering of cutting. Buttherworth Heinemann, Oxford, UK, 2009.

[7] A. Frangi, M. Pagani, U. Perego, and R. Borsari, "Directional cohesive elements for the simulation of blade cutting of thin shells," Computer Modeling in Engineering \& Sciences, vol. 57, no. 3, pp. 205-224, 2010. 
[8] M. Schwarze and S. Reese, "A reduced integration solid-shell finite element based on the EAS and the ANS concept-Large deformation problems," International Journal for Numerical Methods in Engineering, vol. 85, no. 3, pp. 289-329, 2011.

[9] R. W. Macek and B. H. Aubert, "A mass penalty technique to control the critical time increment in explicit dynamic finite element analyses," Earthquake Engng. Struct. Dyn., vol. 24, no. 10, pp. 1315-1331, 1995.

[10] L. Olovsson, K. Simonsson, and M. Unosson, "Selective mass scaling for explicit finite element analyses," Int. J. Numer. Meth. Engng., vol. 63, no. 10, pp. 1436-1445, 2005.

[11] E. Hinton, T. Rock, and O. C. Zienkiewicz, "A note on mass lumping and related processes in the finite element method," Earthquake Engng. Struct. Dyn., vol. 4, no. 3, pp. 245-249, 1976.

[12] L. Olovsson, M. Unosson, and K. Simonsson, "Selective mass scaling for thin walled structures modeled with tri-linear solid elements," Computational Mechanics, vol. 34, no. 2, pp. 134-136, 2004.

[13] F. Abed-Meraim and A. Combescure, "An improved assumed strain solid-shell element formulation with physical stabilization for geometric non-linear applications and elasticplastic stability analysis," International Journal for Numerical Methods in Engineering, vol. 80, no. 13, pp. 1640-1686, 2009.

[14] W. A. Wall, M. Gee, and E. Ramm, "The challenge of a three-dimensional shell formulation: the conditioning problem," in Proc. IASS-IACM 2000, Fourth International Colloquium on Computation for Shells \& Spatial Structures, 2000, pp. 1-21.

[15] M. Gee, E. Ramm, and W. Wall, "Parallel multilevel solution of nonlinear shell structures," Computer Methods in Applied Mechanics and Engineering, vol. 194, no. 21-24, pp. 2513-2533, 2005.

[16] K.-J. Bathe and E. N. Dvorkin, "A four-node plate bending element based on Mindlin/Reissner plate theory and a mixed interpolation," International Journal for $\mathrm{Nu}$ merical Methods in Engineering, vol. 21, no. 2, pp. 367-383, 1985. 